\author{
Wei-Chi Tsai \\ Wei-Wen Chang \\ Yu-Tzu Tsao
}

\section{Obstructive nephropathy presenting as seizures}

Received: 19 December 2014

Accepted: 20 December 2014

Published online: 20 January 2015

(C) Springer-Verlag Berlin Heidelberg and ESICM 2015

W.-C. Tsai and W.-W. Chang contributed equally to this manuscript.

\author{
W.-C. Tsai \\ Department of Medicine, Zuoying Branch Of Kaohsiung Armed \\ Forces General Hospital, Kaohsiung, Taiwan

\section{W.-W. Chang · Y.-T. Tsao} \\ Divison of Nephrology, Department of Medicine, Taoyuan General \\ Hospital, Ministry of Health and Welfare, Taoyuan, Taiwan

\section{Y.-T. Tsao (®)} \\ Department of Critical Care Medicine, Taoyuan General Hospital, \\ Ministry of Health and Welfare, No. 1492, Chung-Shan Road, \\ Taoyuan, Taiwan \\ e-mail: tsaoyutzu@gmail.com \\ Tel.: 886-3-369-9721
}

A 36-year-old woman was referred for the management of generalized tonic-clonic seizure. On admission, episodes of convulsions occurred that shortly turned into status epilepticus, requiring immediate resuscitation and initiation of mechanical ventilation. Although imaging studies of the brain were unremarkable, serum biochemical analyses disclosed high blood urea nitrogen $55.71 \mathrm{mmol} / \mathrm{l}$ (normal range $2.5-8.0 \mathrm{mmol} / \mathrm{l}$ ) and creatinine $689.52 \mu \mathrm{mol} / \mathrm{l}$ (normal range $50-110 \mu \mathrm{mol} / \mathrm{l})$. Nevertheless, she barely responded to intravenous infusion of sodium valproate, lorazepam and phenytoin. Considering the neurological presentations of uremia, hemodialysis was started promptly. After the first two sessions, the epileptic seizures resolved completely. Further workup incriminated a giant retroperitoneal cystic lymphangioma responsible for the postrenal component of uremia (Fig. 1). A complete tumor

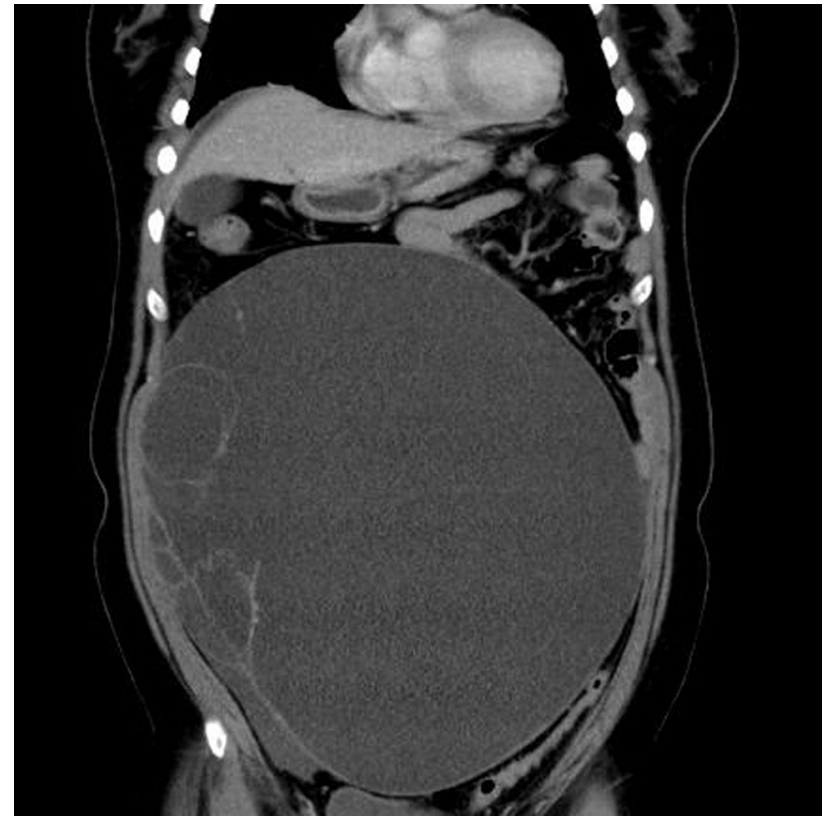

Fig. 1 A coronal reformatted image of contrast-enhanced CT scan showing a large fluid-containing tumor with cystic components occupying the entire abdomen with compression of surrounding structures

resection achieved rapid resolution of obstructive uropathy. The patient was event-free at a 2-year follow-up.

Retroperitoneal cystic lymphangiomas is a rare benign tumor of the lymphatic system, characterized by proliferating lymphatic vessels, slowly growing cysts, and filling with chylous or serous material. It usually presents a diagnostic dilemma, as various types of cystic tumors share similar clinical manifestations. Occasionally, the cystic expansion is large enough to impose on surrounding structures, causing critical compression or obstruction of adjacent retroperitoneal organs. Generally, a total excision is the preferred treatment.

Conflicts of interest None declared. 